\title{
CircParser: a novel streamlined pipeline for circular RNA structure and host gene prediction in non-model organisms
}

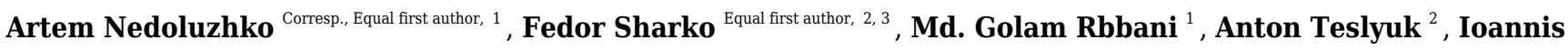 \\ Konstantinidis ${ }^{1}$, Jorge MO Fernandes ${ }^{\text {Corresp. } 1}$ \\ ${ }^{1}$ Faculty of Biosciences and Aquaculture, Nord University, Bodø, Bodø, Norway \\ 2 Complex of NBICS Technologies, National Research Centre "Kurchatov Institute", Moscow, Russia \\ 3 Institute of Bioengineering, Research Center of Biotechnology of the Russian Academy of Sciences, Moscow, Russia, Russia \\ Corresponding Authors: Artem Nedoluzhko, Jorge MO Fernandes \\ Email address: artem.nedoluzhko@nord.no, jorge.m.fernandes@nord.no
}

Circular RNAs (circRNAs) are long noncoding RNAs which play a significant role in various biological processes, including embryonic development and stress responses. These regulatory molecules can modulate microRNA activity and are involved in different molecular pathways as indirect regulators of gene expression. Thousands of circRNAs have been described in diverse taxa due to the recent advances in high throughput sequencing technologies, which led to a huge variety of total RNA sequencing being publicly available. A number of circRNA de novo and host gene prediction tools are available to date, but their ability to accurately predict circRNA host genes is limited in the case of low-quality genome assemblies or annotations. Here, we present CircParser, a simple and fast Unix/Linux pipeline that uses the outputs from the most common circular RNAs in silico prediction tools (CIRI, CIRI2, CircExplorer2, find_circ, and circFinder) to annotate circular RNAs, assigning presumable host genes from local or public databases such as National Center for Biotechnology Information (NCBI). Also, this pipeline can discriminate circular RNAs based on their structural components (exonic, intronic, exon-intronic or intergenic) using genome annotation file. 
1 CircParser: a novel streamlined pipeline for circular RNA structure and host gene 2 prediction in non-model organisms

3

4 Artem Nedoluzhko ${ }^{1, *}$, Fedor Sharko ${ }^{2,3,}{ }^{*}$, Md. Golam Rbbani ${ }^{1}$, Anton Teslyuk ${ }^{3}$, Ioannis

5 Konstantinidis $^{1}$, and Jorge M. O. Fernandes ${ }^{1}$

6

$7{ }^{1}$ Faculty of Biosciences and Aquaculture, Nord University, Bodø, Nordland county, 8 Norway

92 Institute of Bioengineering, Research Center of Biotechnology of the Russian Academy

10 of Sciences, Moscow, Russia

$11{ }^{3}$ Complex of NBICS Technologies, National Research Centre "Kurchatov Institute",

12 Moscow, Russia

Corresponding Author:

Artem Nedoluzhko ${ }^{1}$

Universitetsalléen 11, Bodø, Nordland county, 8049, Norway

Email address: artem.nedoluzhko@nord.no

Jorge M. O. Fernandes ${ }^{1}$

Universitetsalléen 11, Bodø, Nordland county, 8049, Norway

Email address: jorge.m.fernandes@nord.no

\section{Abstract}

Circular RNAs (circRNAs) are long noncoding RNAs which play a significant role in various biological processes, including embryonic development and stress responses. These regulatory molecules can modulate microRNA activity and are involved in different molecular pathways as indirect regulators of gene expression. Thousands of circRNAs have been described in diverse taxa due to the recent advances in high throughput sequencing technologies, which led to a huge variety of total RNA sequencing being publicly available. A number of circRNA de novo and host gene prediction tools are available to date, but their ability to accurately predict circRNA host genes is limited in the case of low-quality genome assemblies or annotations. Here, we present CircParser, a simple and fast Unix/Linux pipeline that uses the outputs from the most common circular RNAs in silico prediction tools (CIRI, CIRI2, CircExplorer2, find_circ, and circFinder) to annotate circular RNAs, assigning 
35

36

37

38

39

40

41

42

43

44

45

46

47

48

49

50

51

52

53

54

55

56

57

58

59

60

61

62

63

64

65

66

67

68

presumable host genes from local or public databases such as National Center for Biotechnology Information (NCBI). Also, this pipeline can discriminate circular RNAs based on their structural components (exonic, intronic, exon-intronic or intergenic) using genome annotation file.

\section{Introduction}

De novo genome sequencing has become a routine procedure, due to a decrease in sequencing costs, diversification of high-throughput sequencing platforms and improvement of bioinformatic tools (Ekblom \& Wolf 2014). However, the quality of non-model species genome assemblies and, as a result, their annotations are often of unsatisfactory quality, because of (1) repetitive sequences, including transposons, and short sequence repeats (SSRs); (2) gene and genome duplications; (3) single-nucleotide polymorphisms (SNPs) and genome rearrangements (Lien et al. 2016; Negrisolo et al. 2010; Rodriguez \& Arkhipova 2018; Yahav \& Privman 2019).

CircRNAs are relatively poorly studied members of the non-coding RNA family. These unique single-stranded molecules are generated through back-splicing of pre-mRNAs in a wide range of eukaryotic and prokaryotic taxa (Danan et al. 2012; Holdt et al. 2018), and even viruses (Huang et al. 2019). CircRNAs play a significant role in the regulation of the molecular pathways not only through modulating of microRNA and protein activity, but also by the affecting transcription or splicing (Holdt et al. 2018).

These regulatory molecules have been known for decades, but the development of highthroughput DNA analysis methods lead to a rapid increase in the number of studies related to these type of non-coding RNAs. This, in turn, resulted in a requirement for additional circRNA prediction tools. The miARma-Seq (Andres-Leon \& Rojas 2019) with CIRI predictor (Gao et al. 2015), circRNA_finder (Westholm et al. 2014), find_circ (Memczak et al. 2013), CIRCexplorer2 (Zhang et al. 2016), and other tools are very popular today for prediction of circRNAs sequences based on transcriptomic data (Hansen et al. 2016; Szabo \& Salzman 2016), despite significant output differences. Several circRNA predictors (CIRI, CIRI2, and CircExplorer2) can use genome annotation files for host gene prediction but they are definitely useful only for wellannotated genomes, and even, such as CircView (Feng et al. 2018) or circMeta (Chen et al. 2019), have been designed specifically for them.

7 Here we describe CircParser, a novel and easy to use Unix/Linux pipeline for prediction 8 of host gene circular RNAs using the blastn program and the freely available bedtools 
69 software (Quinlan \& Hall 2010). CircParser can be also implemented as a part of 70 pipelines for de novo prediction of circular RNA because of its versatile output files.

71 CircParser is most useful for circRNA host gene prediction analysis in whole 72 transcriptomic datasets for low-quality assembled, as well as poorly annotated 73 genomes. It sorts and joins overlapped circular RNAs sequences and predicts host gene 74 name for overrepresented circRNAs, while identifying their structural components. We 75 demonstrate the prediction capacity of CircParser on a recently published 76 transcriptomic data set from the wild and domesticated females of Nile tilapia 77 (Oreochromis niloticus) fast muscle (Konstantinidis et al., under review) using the five 78 most popular circRNAs in silico prediction tools - CIRI, CIRI2, CircExplorer2, find_circ, 79 and circFinder.

80

81

82

83

84

85

86

87

88

89

90

91

92

93

94

95

96

97

98

99

100

101

102

\section{Materials \& Methods}

The results of Illumina sequencing of twelve ribosomal RNA depleted RNA-seq libraries reads have been downloaded from Gene Expression Omnibus (accession number GSE135811). The DNA reads were filtered by quality (phred $>20$ ) and library adapters were trimmed using Cutadapt software (version 1.12) (Marcel 2011). The Nile tilapia reference genome (ASM185804v2) and its gene-annotation (ref_O_niloticus_UMD_NMBU_top_level.gff3) were used in the following analysis. CircRNA prediction was performed for each ribosomal RNA depleted RNA-seq library using the circRNA in silico prediction tools i) CIRI (Gao et al. 2015) that is linked to miARma-Seq pipeline (Andres-Leon \& Rojas 2019), ii) CIRI2 (Gao et al. 2018), iii) CircExplorer2 (Zhang et al. 2016), iv) find_circ (Memczak et al. 2013), and v) circFinder (Westholm et al. 2014). Prediction output files from all libraries were converted separately to coordinate file format. After sorting, these coordinate files (from different prediction algorithms, but for each library) were merged using bedtools multiinter (Quinlan \& Hall 2010) to determine a joint prediction output from CIRI, CIRI2, CircExplorer2, find_circ, and circFinder (see Supplementary Table S1).

We developed CircParser, as a streamlined pipeline, which makes use output files from the most popular circRNAs in silico predictors. CircParser only works under Linux/Unix system. The parameters for CircParser are presented in Table 1.

Usage: perl CircParser.pl [-h] -b INPUT_FILE-genome REF_GENOME 
103 [Table 1]

104

105 CircParser can merge overlapped circRNAs coordinates from circRNAs predictor

106 outputs using bedtools merge (Quinlan \& Hall 2010) at the first stage of the pipeline;

107 this ensures that they are related to the same host gene and creates separate coordinates

108 files (bed file) with overlapped circRNAs coordinates. In addition, it is optionally

109 possible to merge circRNA without overlapping coordinates but located in the

110 contiguous genome locus using the special option.

111 The separate coordinate files (bed file) are converted to fasta files using bedtools

112 getfasta (Quinlan \& Hall 2010). Finally, CircParser uses fasta files for host gene

113 prediction using a NCBI database (the longest stage of pipeline) for circRNAs (Figure

114 1A). CircParser works by default with the NCBI online database, but it can optionally

115 use a custom database or a pre-compiled NCBI database installed locally. CircParser

116 includes the following blast parameters, which are necessary for host gene prediction,

117 and assigns sequences to the respective circRNA: -perc_identity 90; -max_target_seqs 1000;

118 -max_hsps 1; the maximum number of aligned sequences to keep is 1000; the minimum

119 percent identity of matches to report is $90 \%$. CircParser also filters out non-informative

120 blast results, such as "uncharacterized", "clone", "linkage group" and others from the

121 output table.

122

123 [Figure 1]

124

125 CircParser can also discriminate circular RNAs by their structural components: exonic,

126 intronic, exon-intronic or intergenic using genome annotation gff/gff3 file (-a

127 parameter). In this case, the user should avoid circRNAs coordinate merging (using --

128 np parameter) during the pipeline implementation for correct results (Figure 1B).

129 Usage: perl CircParser.pl -np -b INPUT_FILE -genome REF_GENOME -a GENOME.gff

130 However, poor quality of annotation file can lead to errors in the circRNAs structure

131 analysis.

132 The Perl implementation of CircParser is available at

133 https://github.com/SharkoTools/CircParser

134

135

Results

Peer) reviewing PDF | (2019:12:43636:1:1:CHECK 23 Jan 2020) 
136 We applied CircParser to twelve merged coordinate files that contained information 137 about joint coordinates for circRNAs predicted using CircExplorer2, miARma-Seq (with 138 CIRI predictor), CIRI2, find_circ, and circFinder. The five different algorithms predicted 139 on average 131 (CircExplorer2); 501 (CIRI); 706 (CIRI2); 257 (find_circ), and 398 140 (circFinder) circRNAs per sample, with an insignificant overlap $\sim 37$ circRNAs (Figure 141 2; Supplementary Table S1), similarly to previously published comparisons (Hansen 142 2018; Hansen et al. 2016).

144 [Figure 2]

146 To access the host gene of circular RNAs and to reduce false-positive rates, only 147 overlapping circRNAs (Figure 2) were used in CircParser. This pipeline allows the 148 elimination of non-informative outputs (e.g contains only chromosome/contig name, 149 number of uncharacterized loci, or name of BAC clone, and etc.), while keeping more 150 the relevant blast results and retrieving the likely host gene name for the circular RNAs; 151 in the case of impossibility to find identical sequences in the database, this tool mark 152 these sequence as NOT ASSIGNED).

153

154

\section{Discussion}

The CircParser results also allow to determine the number of circRNA types from one host gene and their minimum and maximum size in base pairs (bp). We showed that our algorithm detected presumable host gene names for the vast majority of predicted circRNAs. Moreover, most of them were related to muscle functions (e.g. calcium/calmodulin-dependent protein kinase, troponin T3, myocyte-specific enhancer factor $2 \mathrm{C}$, and others), and immune-related genes (MHC class IA antigen), which were consistently found among different individuals (Supplementary Table S2), despite the relatively low coverage for circRNAs analysis of the sequencing data used (Mahmoudi \& Cairns 2019). An example of circRNA structure analysis for CIRI, CIRI2, CircExplorer2, find_circ, and circFinder outputs are presented in Supplementary Table S3.

To estimate the capacity of our pipeline we compared a number of host genes that were predicted by CircExplorer2 and CircParser (CircExplorer2 outputs were used as input files) for the same O. niloticus fast muscle datasets used earlier. As a result, CircParser shows greater efficiency for Nile tilapia, improving the number of predicted host genes up to two-fold (Figure 3). 
170

171 [Figure 3]

172

173 Another equally important aspect of CircParser concerned the accuracy of this pipeline.

174 The most well-annotated reference genome of zebrafish (assembly GRCz11) and

175 zebrafish muscle transcriptomic dataset (ERR145655) were used for accuracy

176 estimation, i.e. the agreement between the annotation file and CircParser output. We

177 showed that in this case, CircParser host gene prediction was confirmed in $82.4 \%$ cases.

178

179

180

181

182

183

184

185

186

187

188

189

190

191

192

193

194

195

196

197

198

199

200

201

202

203

\section{Conclusions}

Thus, we conclude that CircParser represents a reproducible workflow that enables researchers to effectively predict the host genes for circular RNAs, even in non-model organisms with poorly annotated genome assemblies.

\section{Acknowledgements}

We would like to acknowledge Jorge Galindo-Villegas from Nord University (Norway) and Tomas B. Hansen from the Aarhus University (Denmark) for their valuable advice.

\section{References}

Andres-Leon E, and Rojas AM. 2019. miARma-Seq, a comprehensive pipeline for the simultaneous study and integration of miRNA and mRNA expression data. Methods 152:31-40. 10.1016/j.ymeth.2018.09.002

Chen L, Wang F, Bruggeman EC, Li C, and Yao B. 2019. circMeta: a unified computational framework for genomic feature annotation and differential expression analysis of circular RNAs. Bioinformatics. 10.1093/bioinformatics/btz606

Danan M, Schwartz S, Edelheit S, and Sorek R. 2012. Transcriptome-wide discovery of circular RNAs in Archaea. Nucleic Acids Res 40:3131-3142. 10.1093/nar/gkr1009

Ekblom R, and Wolf JB. 2014. A field guide to whole-genome sequencing, assembly and annotation. Evol Appl 7:1026-1042. 10.1111/eva.12178 
204 Feng J, Xiang Y, Xia S, Liu H, Wang J, Ozguc FM, Lei L, Kong R, Diao L, He C, and Han

205

206

207

208

209

210

211

212

213

214

215

216

217

218

219

220

221

222

223

224

225

226

227

228

229

230

231

232

233

234

235

236

237

238

239

240

241

242

L. 2018. CircView: a visualization and exploration tool for circular RNAs. Brief Bioinform 19:1310-1316. 10.1093/bib/bbx070

Gao Y, Wang J, and Zhao F. 2015. CIRI: an efficient and unbiased algorithm for de novo circular RNA identification. Genome Biol 16:4. 10.1186/s13059-014-0571-3

Gao Y, Zhang J, and Zhao F. 2018. Circular RNA identification based on multiple seed matching. Brief Bioinform 19:803-810. 10.1093/bib/bbx014

Hansen TB. 2018. Improved circRNA Identification by Combining Prediction Algorithms. Front Cell Dev Biol 6:20. 10.3389/fcell.2018.00020

Hansen TB, Veno MT, Damgaard CK, and Kjems J. 2016. Comparison of circular RNA prediction tools. Nucleic Acids Res 44:e58. 10.1093/nar/gkv1458

Holdt LM, Kohlmaier A, and Teupser D. 2018. Molecular roles and function of circular RNAs in eukaryotic cells. Cell Mol Life Sci 75:1071-1098. 10.1007/s00018-017-26885

Huang JT, Chen JN, Gong LP, Bi YH, Liang J, Zhou L, He D, and Shao CK. 2019. Identification of virus-encoded circular RNA. Virology 529:144-151. 10.1016/j.virol.2019.01.014

Lien S, Koop BF, Sandve SR, Miller JR, Kent MP, Nome T, Hvidsten TR, Leong JS, Minkley DR, Zimin A, Grammes F, Grove H, Gjuvsland A, Walenz B, Hermansen RA, von Schalburg K, Rondeau EB, Di Genova A, Samy JK, Olav Vik J, Vigeland MD, Caler L, Grimholt U, Jentoft S, Vage DI, de Jong P, Moen T, Baranski M, Palti Y, Smith DR, Yorke JA, Nederbragt AJ, Tooming-Klunderud A, Jakobsen KS, Jiang X, Fan D, Hu Y, Liberles DA, Vidal R, Iturra P, Jones SJ, Jonassen I, Maass A, Omholt SW, and Davidson WS. 2016. The Atlantic salmon genome provides insights into rediploidization. Nature 533:200-205. 10.1038/nature17164

Mahmoudi E, and Cairns MJ. 2019. Circular RNAs are temporospatially regulated throughout development and ageing in the rat. Sci Rep 9:2564. 10.1038/s41598019-38860-9

Marcel M. 2011. Cutadapt Removes Adapter Sequences From High-Throughput Sequencing Reads. EMBnetjournal 17:10-12. 10.14806/ej.17.1.200.

Memczak S, Jens M, Elefsinioti A, Torti F, Krueger J, Rybak A, Maier L, Mackowiak SD, Gregersen LH, Munschauer M, Loewer A, Ziebold U, Landthaler M, Kocks C, le Noble F, and Rajewsky N. 2013. Circular RNAs are a large class of animal RNAs with regulatory potency. Nature 495:333-338. 10.1038/nature11928

Negrisolo E, Kuhl H, Forcato C, Vitulo N, Reinhardt R, Patarnello T, and Bargelloni L. 2010. Different phylogenomic approaches to resolve the evolutionary relationships among model fish species. Mol Biol Evol 27:2757-2774. 10.1093/molbev/msq165

Peer) reviewing PDF | (2019:12:43636:1:1:CHECK 23 Jan 2020) 
243 Quinlan AR, and Hall IM. 2010. BEDTools: a flexible suite of utilities for comparing 244 genomic features. Bioinformatics 26:841-842. 10.1093/bioinformatics/btq033

245 Rodriguez F, and Arkhipova IR. 2018. Transposable elements and polyploid evolution 246 in animals. Curr Opin Genet Dev 49:115-123. 10.1016/j.gde.2018.04.003

247 Szabo L, and Salzman J. 2016. Detecting circular RNAs: bioinformatic and experimental $248 \quad$ challenges. Nat Rev Genet 17:679-692. 10.1038/nrg.2016.114

249 Westholm JO, Miura P, Olson S, Shenker S, Joseph B, Sanfilippo P, Celniker SE, 250 Graveley BR, and Lai EC. 2014. Genome-wide analysis of drosophila circular RNAs reveals their structural and sequence properties and age-dependent neural accumulation. Cell Rep 9:1966-1980. 10.1016/j.celrep.2014.10.062

Yahav T, and Privman E. 2019. A comparative analysis of methods for de novo assembly of hymenopteran genomes using either haploid or diploid samples. Sci Rep 9:6480. 10.1038/s41598-019-42795-6

Zhang XO, Dong R, Zhang Y, Zhang JL, Luo Z, Zhang J, Chen LL, and Yang L. 2016. Diverse alternative back-splicing and alternative splicing landscape of circular RNAs. Genome Res 26:1277-1287. 10.1101/gr.202895.115 


\section{Table $\mathbf{1}$ (on next page)}

Table 1. CircParser.pl usage. Required and optional parameters

Table 1. CircParser.pl usage. Required and optional parameters 
1 Table 1. CircParser.pl usage. Required and optional parameters

Parameter

-h, --help

-b

-g, --genome

$-t,-\operatorname{tax}$

$-\mathbf{a}$

$--n p$

$-c,-$ ciri

--threads

-v, --version

\section{Parameter description}

Show this help message and exit

CircRNA input file (required)

Reference genome file (required)

NCBI TaxID (optional)

Genome annotation file, gff/gff3 file (optional)

Prohibition for coordinate merging (optional)

Input circRNA from CIRI|CIRI2 in silico predictors, (default: input

from CircExplorer2, find_circ, circFinder, and BED files)

Number of threads (CPUs) for BLAST search (optional)

Current CircParser version 


\section{Figure 1}

An overview of the CircParser pipeline

An overview of the CircParser pipeline: Figure 1A: The pipeline includes merging of the circRNAs with overlapping genome coordinates and presents the number of different circRNAs originating from one host gene. Figure 1B: CircParser includes the prediction of circRNA structural components using a genome annotation gff/gff3 file

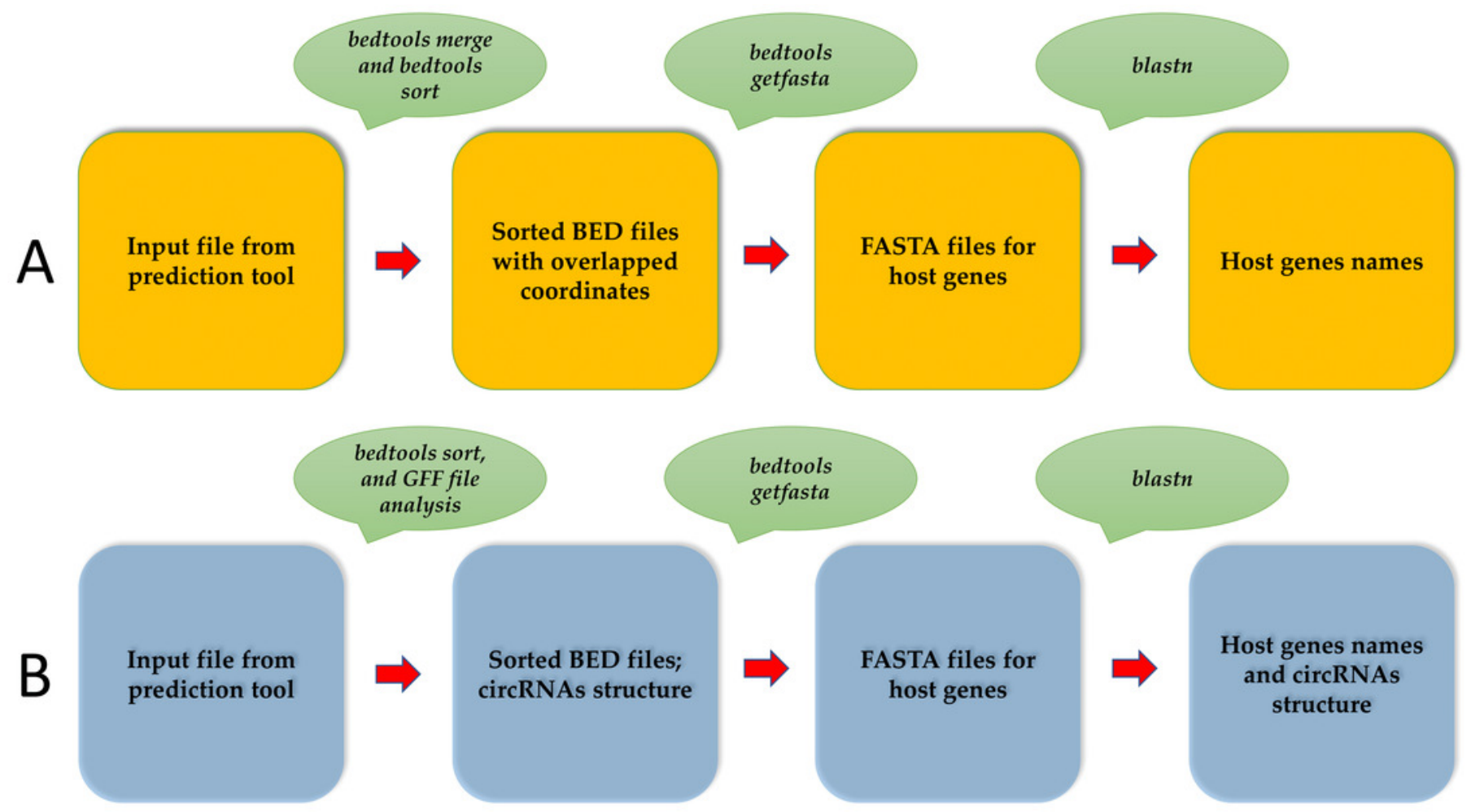


Figure 2

Number of circular RNAs that have been predicted by CIRI, CIRI2, CircExplorer2, find_circ, circFinder, and that are common between all prediction algorithms

Number of circular RNAs that have been predicted by CIRI, CIRI2, CircExplorer2, find_circ, circFinder, and that are common between all prediction algorithms

Overlapping cirularRNAs from five predictors

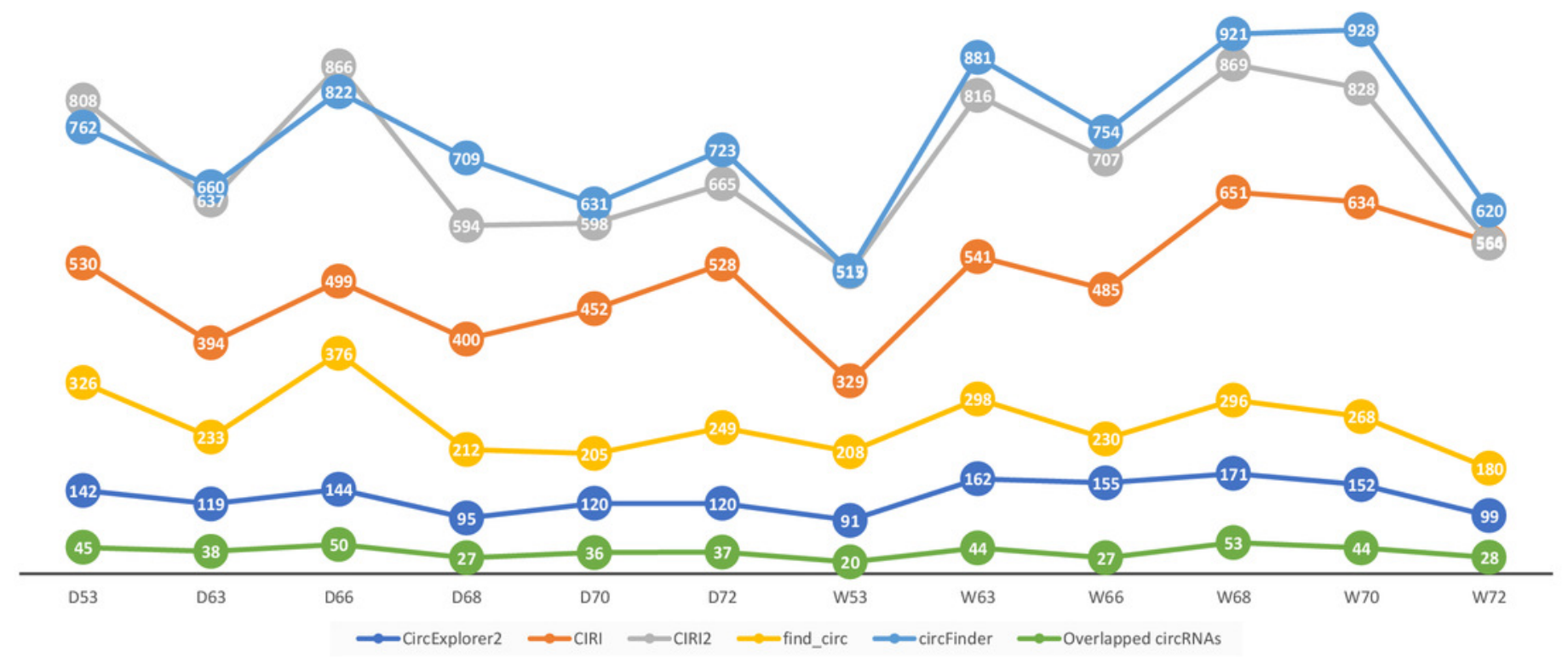




\section{Figure 3}

CircParser capacity: number of host genes that were predicted by CircExplorer2 and CircParser

CircParser capacity: number of host genes that were predicted by CircExplorer2 and CircParser

\section{The number of predicted host genes for Nile tilapia datasets}

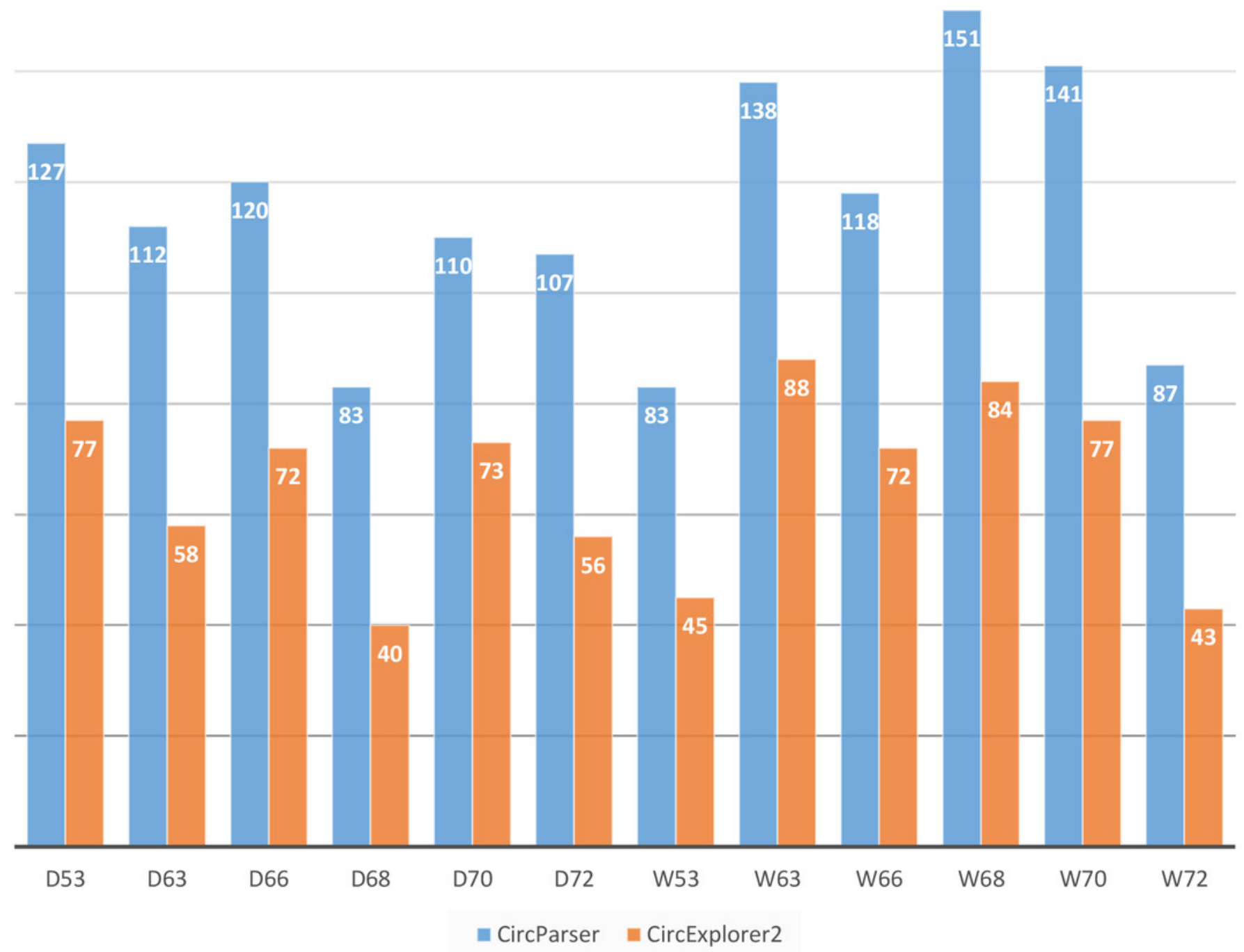

